\title{
Neurosyphilis se manifestant par de l'hypomanie
}

\author{
Caroline Jeon MD, Kevin Gough MD MÉd
}

Citation : CMAJ 2021 August 3;193:E1177. doi : 10.1503/cmaj.201892-f

Voir la version anglaise de l'article ici : www.cmaj.ca/lookup/doi/10.1503/cmaj.201892

$\mathbf{U}$ n homme de 60 ans vivant avec le VIH a consulté son médecin de famille pour des problèmes de concentration depuis 3 mois. Au cours des 2 dernières semaines, il a remarqué un érythème étendu et indolore au torse, au dos et aux extrémités (figure 1). Il a été consommateur de méthamphétamine en crystaux, mais pas récemment. Il déclarait avoir eu d'occasionnelles relations sexuelles non protégées avec des hommes et des femmes. Il était difficile de suivre le fil de ses pensées; son débit était rapide, il semblait distrait et formulait des plans grandioses et irréalistes, en contradiction avec sa situation actuelle. Il a perdu son entreprise et son logis. Sa numération des lymphocytes T CD4 était de 159 (488-1711) cellules/ $\mu \mathrm{L}$ ou $0,16(0,49-$ $1,71) \times 10^{9} / \mathrm{L}$ et sa charge virale du VIH était de 301108 copies d'ARN/mL.

La présence d'érythème, les relations sexuelles non protégées et les changements comportementaux nous ont fait soupçonner la neurosyphilis infectieuse avec hypomanie. Nous avons diagnostiqué une syphilis secondaire sur la base d'un résultat positif au dépistage du tréponème pâle au moyen d'un test d'immunodosage sérique par chimioluminescence (CMIA), d'un taux élevé au test rapide de la réagine plasmatique (RPR), à 1:64, et d'un résultat positif à l'épreuve d'agglutination des particules de tréponème pâle (TP-PA). L'examen du liquide céphalora- chidien, également nécessaire pour poser le diagnostic de neurosyphilis, a montré un taux élevé de protéines, à 1,01 (éventail des valeurs normales $0,15-0,45$ ) g/L, et un VDRL (Venereal Disease Research Laboratory) positif, avec un titre de 1:1. Nous avons traité notre patient au moyen de pénicilline intraveineuse ( 4 millions d'unités toutes les $4 \mathrm{~h}$ pendant $14 \mathrm{j}$ ).

Le taux de syphilis est en hausse au Canada depuis une dizaine d'années ${ }^{1}$. Les éclosions affectent souvent de manière disproportionnée les hommes ayant des relations sexuelles avec d'autres hommes, même si certaines se sont aussi déclarées chez des personnes hétérosexuelles ces dernières années, en zones urbaines et rurales ${ }^{1,2}$. La neurosyphilis est

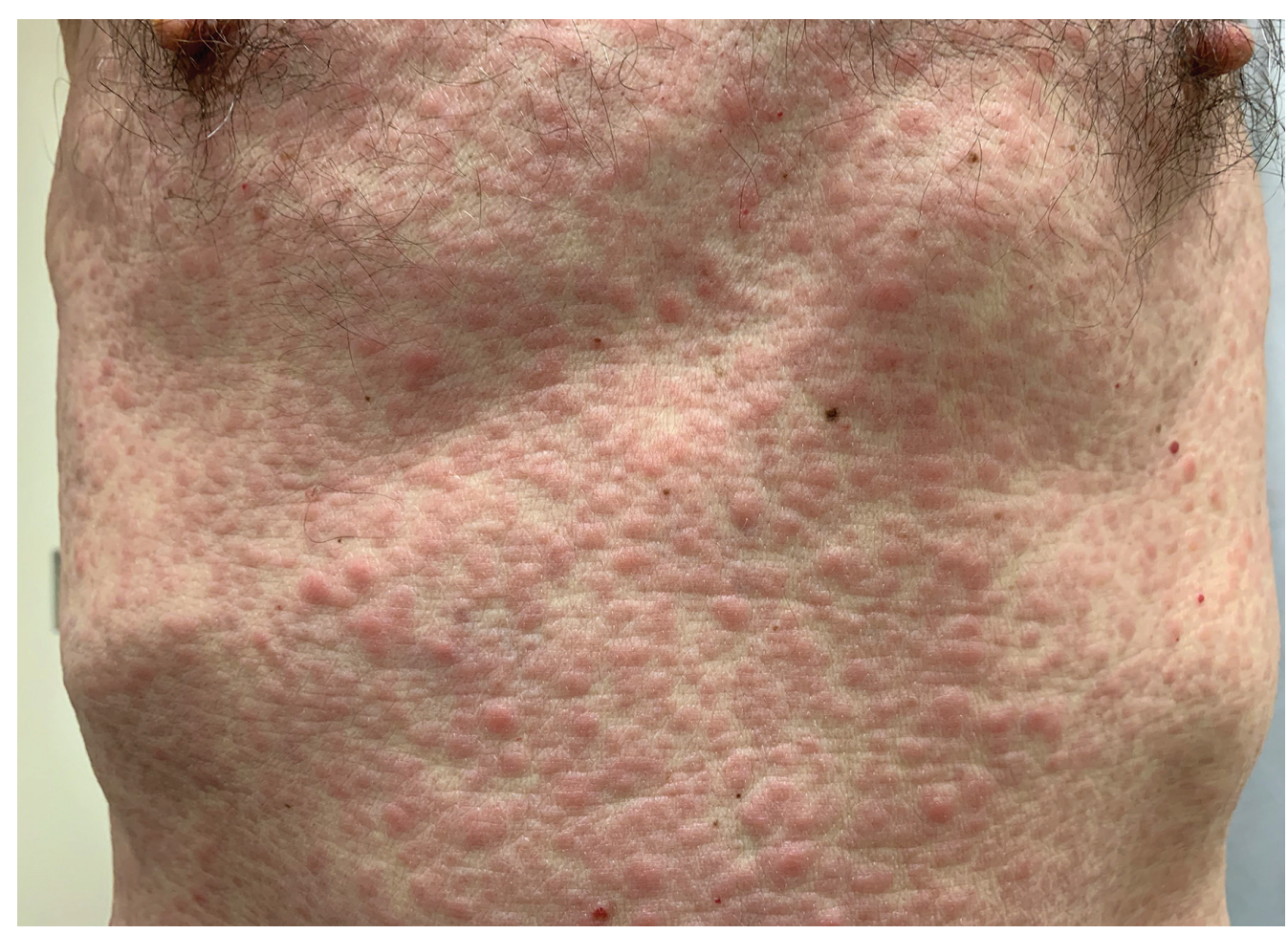

Figure 1 : Photographie du torse d'un homme de 60 ans atteint de syphilis. L'érythème n'était ni douloureux ni prurigineux et s'étendait aux 2 bras et aux 2 jambes. 
plus fréquente chez les personnes concomitamment porteuses du VIH, surtout quand elles ont une numération des T CD4 inférieure à 350 cellules $/ \mu \mathrm{L}\left(0,35 \times 10^{9} / \mathrm{L}\right)^{3}$. Selon des études rétrospectives, $40 \%$ des patients présentent une atteinte oculaire, incluant l'uvéite, la rétinopathie et la perte visuelle. Un tiers des patients ont une atteinte cognitive et un tiers ont des manifestations neurologiques, telles que céphalées, aphasie, hallucinations auditives et $\mathrm{AVC}^{4,5}$. Le cas échéant, les symptômes psychiatriques varient beaucoup. L'hypomanie est rare, affectant moins de $10 \%$ des personnes atteintes de neurosyphilis ${ }^{4}$. Les séquelles neurologiques sont souvent permanentes, mais notre patient s'est tout à fait rétabli après son traitement de pénicilline intraveineuse administré en clinique externe ${ }^{3}$.

\section{Références}

1. Report on sexually transmitted infections in Canada: 2013-2014. Ottawa: Public Health Agency of Canada; 2017. Accessible ici : https://www.canada. $\mathrm{ca} /$ content/dam/phac-aspc/documents/services/publications/diseases -conditions/report-sexually-transmitted-infections-canada-2013-14/report -sexually-transmitted-infections-canada-2013-14-eng.pdf (consulté le 18 mai 2020).

2. Syphilis: key information and resources. Ottawa: Public Health Agency of Canada; 2020. Accessible ici : https://www.canada.ca/en/public-health/ services/infectious-diseases/sexual-health-sexually-transmitted-infections/ canadian-guidelines/syphilis.html (consulté le 30 nov. 2020).

3. WA Lynn, Lightman S. Syphilis and HIV: a dangerous combination. Lancet Infect Dis 2004;4:456-66.

4. Landry $\mathrm{T}$, Smyczek $\mathrm{P}$, Cooper R, et al. Retrospective review of tertiary and neurosyphilis cases in Alberta, 1973-2017. BMJ Open 2019;9:e025995.

5. Lin L-R, Zhang H-L, Huang S-J, et al. Psychiatric manifestations as primary symptom of neurosyphilis among HIV-negative patients. J Neuropsychiatry Clin Neurosci 2014;26:233-40.

Intérêts concurrents : Aucun déclaré.

Cet article a été révisé par des pairs.

Les auteurs ont obtenu le consentement du patient.

Affiliations : Département de médecine familiale et communautaire (Jeon) et Division d'infectiologie (Gough), Département de médecine, Hôpital St. Michael, Université de Toronto, Toronto, Ont.

Propriété intellectuelle du contenu : Il s'agit d'un article en libre accès distribué conformément aux modalités de la licence Creative Commons Attribution (CC BY-NC-ND 4.0), qui permet l'utilisation, la diffusion et la reproduction de tout médium à la condition que la publication originale soit adéquatement citée, que l'utilisation se fasse à des fins non commerciales (c.-à-d., recherche ou éducation) et qu'aucune modification ni adaptation n'y soit apportée. Voir : https://creativecommons.org/licenses/by-nc-nd/4.0/deed.fr.

Correspondance : Caroline Jeon, caroline.jeon@unityhealth.to

Les images cliniques sont choisies pour leur caractère particulièrement intéressant, classique ou impressionnant. Toute soumission d'image de haute résolution claire et bien identifiée doit être accompagnée d'une légende aux fins de publication. On demande aussi une brève explication (300 mots maximum) de la portée éducative des images, et des références minimales. Le consentement écrit du patient au regard de la publication doit être obtenu avant la soumission. 